\title{
Autism and measles, mumps, and rubella vaccine: no epidemiological evidence for a causal association
}

\author{
Brent Taylor, Elizabeth Miller, C Paddy Farrington, Maria-Christina Petropoulos, Isabelle Favot-Mayaud, Jun Li, \\ Pauline A Waight
}

\section{Summary}

Background We undertook an epidemiological study to investigate whether measles, mumps, and rubella (MMR) vaccine may be causally associated with autism.

Methods Children with autism born since 1979 were identified from special needs/disability registers and special schools in eight North Thames health districts, UK. Information from clinical records was linked to immunisation data held on the child health computing system. We looked for evidence of a change in trend in incidence or age at diagnosis associated with the introduction of MMR vaccination to the UK in 1988. Clustering of onsets within defined postvaccination periods was investigated by the case-series method.

Findings We identified 498 cases of autism (261 of core autism, 166 of atypical autism, and 71 of Asperger's syndrome). In 293 cases the diagnosis could be confirmed by the criteria of the International Classification of Diseases, tenth revision (ICD10: 214 [82\%] core autism, 52 [31\%] atypical autism, 27 [38\%] Asperger's syndrome). There was a steady increase in cases by year of birth with no sudden "step-up" or change in the trend line after the introduction of MMR vaccination. There was no difference in age at diagnosis between the cases vaccinated before or after 18 months of age and those never vaccinated. There was no temporal association between onset of autism within 1 or 2 years after vaccination with MMR (relative incidence compared with control period 0.94 [95\% $\mathrm{Cl} 0.60-1.47]$ and 1.09 [0.79-1.52]). Developmental regression was not clustered in the months after vaccination (relative incidence within 2 months and 4 months after MMR vaccination 0.92 [0.38-2.21] and 1.00 [0.52-1.95]). No significant temporal clustering for age at onset of parental concern was seen for cases of core autism or atypical autism with the exception of a single interval within 6 months of MMR vaccination. This appeared to be an artifact related to the difficulty of defining precisely the onset of symptoms in this disorder.

Interpretation Our analyses do not support a causal association between MMR vaccine and autism. If such an association occurs, it is so rare that it could not be identified in this large regional sample.

Lancet 1999; 353: 2026-29

See Commentary page 1987

Department of Community Child Health, Royal Free Campus, Royal Free and University College Medical School, University College London, London NW3 2QG, UK (Prof B Taylor FRCPCH, M-C Petropoulos MRCP, I Favot-Mayaud MD, J Li PhD); Immunisation Division, Public Health Laboratory Service Communicable Disease Surveillance Centre, London (E Miller FRCPath, P A Waight BSc); and Department of Statistics, Open University (C P Farrington PhD)

Correspondence to: Prof Brent Taylor

\section{Introduction}

Wakefield and colleagues ${ }^{1}$ postulated that measles, mumps, and rubella (MMR) vaccination might be causally linked with autism. Although there is no scientific evidence to support this claim, ${ }^{2-4}$ neither are there robust data on the prevalence of autism in children born before and after the introduction of MMR vaccine to the UK in 1988. The postulated causal link between MMR vaccination and autism was based on a reported close temporal association between these two events. ${ }^{1}$ Since MMR vaccine is given at around $12-15$ months of age and the mean age at which parents of children with autism first report concern about their child's development is $18-19$ months, ${ }^{5}$ a close temporal association in some autistic children would be expected by chance. ${ }^{6}$

We undertook a population-based study in the North East Thames region to investigate trends in the incidence of autistic disorders before and after the introduction of MMR vaccine in October, 1988, and the immunisation histories of children with these disorders. We used caseseries analysis methods to test for clustering of onsets within defined postvaccination periods.

\section{Patients and methods}

Children with autistic disorders born since 1979 were identified in eight health districts in mid-1998 from computerised special needs/disability registers at child development centres and from records in special schools. Information on children with such disorders who were younger than 16 years of age was extracted from clinical records by one of three experienced paediatric registrars. The information extracted included the age at which the autistic disorder was diagnosed, the recorded age at which the parents first became concerned about the child's developmental state, and the age at which the regression became obvious, if that was a feature.

By use of criteria of the International Classification of Diseases, tenth revision (ICD10), the diagnosis of autism was checked against information in the available records on the child's present condition and his or her condition between the ages of 18 months and 3 years. Study investigators worked in pairs with opportunity for discussion to reach consensus when there was ambiguity. Inter-rater reliability was tested on 20 case records (independent completion of the data-collection form); the concordance was above $95 \%$. Immunisation data, which were recorded independently of the clinical record, with exact dates, were obtained from the Regional Interactive Child Health Computing System (RICHS).

Three statistical analyses were undertaken. First, trends in the time series of cases were analysed by Poisson regression. Because of delays in diagnosis, ascertainment of cases in later years is incomplete. To circumvent this problem, only cases aged 0-59 months at diagnosis and born in the years 1979-92 were included in this analysis. We looked for evidence of a change after 1987, first by allowing a "step-up" in the 1987 and later birth cohorts and second by allowing the exponential trends to differ before and after 1987 .

Second, the age at diagnosis was compared in vaccinated and unvaccinated children with autism diagnosed after the age of 


\begin{tabular}{|c|c|c|c|c|c|c|}
\hline \multirow[t]{2}{*}{ Variable } & \multicolumn{2}{|c|}{$\begin{array}{l}\text { Core autism } \\
(n=261)\end{array}$} & \multicolumn{2}{|c|}{$\begin{array}{l}\text { Atypical } \\
\text { autism }(n=166)\end{array}$} & \multicolumn{2}{|c|}{$\begin{array}{l}\text { Asperger's } \\
\text { syndrome }(n=71)\end{array}$} \\
\hline & $\mathrm{n}$ & $\begin{array}{l}\text { Median } \\
\text { (months) }\end{array}$ & $\mathrm{n}$ & $\begin{array}{l}\text { Median } \\
\text { (months) }\end{array}$ & $\mathrm{n}$ & $\begin{array}{l}\text { Median } \\
\text { (months) }\end{array}$ \\
\hline Age at diagnosis & 235 & 37 & 122 & 42 & 67 & 73 \\
\hline Age at parental concern & 207 & 19 & 119 & 21 & 48 & 24 \\
\hline Age at regression & 75 & 18 & 30 & 18 & 4 & 30 \\
\hline $\begin{array}{l}\text { Interval concern to } \\
\text { diagnosis }\end{array}$ & 235 & 22 & 122 & 26 & 67 & 53 \\
\hline $\begin{array}{l}\text { Interval regression to } \\
\text { diagnosis }\end{array}$ & 73 & 17 & 27 & 17 & 4 & 14.5 \\
\hline
\end{tabular}

$\mathrm{n}=$ number of cases for whom information was available.

Table 1: Median (in elapsed months of age) for age at

diagnosis, age at parental concern, and age at regression, and intervals between these, according to diagnostic category

18 months. Children were classified into three categories: those who had received MMR vaccine before the age of 18 months; those never vaccinated with MMR; and those who had received MMR vaccine at age 18 months or later. Because of the skewed distribution of the age at diagnosis of autism, the analysis was done on logarithms of age, with linear regression to compare the mean log ages in the three vaccine categories, and with control for the effect of birth cohort.

Third, possible temporal associations between vaccinations and the age at diagnosis of autism, the recorded age at parental concern, and the age of onset of regression were analysed by the case-series method. ${ }^{7-9}$ This method is valid for rare chronic disorders of acute onset. For autism diagnosis, we investigated periods within 1 or 2 years after vaccination as the risk periods. For date at parental concern, we looked at periods of within 6 months or 1 year after vaccination. Because of the suggestion that regression may be an acute event after vaccination ${ }^{1}$ we considered periods of within 2 months, 4 months, and 6 months of vaccination. Where vaccination and the event of interest occurred in the same month, we assumed that vaccination preceded the event. Two analyses were done for each combination of endpoint and risk period; the first took into account only MMR vaccine, with single-antigen measles vaccine and combined mumps and rubella vaccine ignored; and the second included all three types of vaccine. In each analysis, the reference period for each individual consisted of every month from birth to the end of August, 1998, that did not fall during a postvaccination risk period. All analyses were finely stratified for age, particularly in younger age-groups, because of the multimodal age distribution of recorded events. 17 age-groups were used for autism diagnosis, 30 for parental concern, and 21 for regression.

\section{Results}

498 children with autism were identified: 261 with typical (core) autism (prevalence rate in children under 16 years of age 5.3 per 10000$), 166(3.4$ per 10000$)$ with atypical autism, and $71(1.4$ per 10000$)$ with Asperger's syndrome. The diagnosis could be confirmed with ICD10 criteria, from information recorded in the clinical notes, in $214(82 \%)$ cases of core autism, $52(31 \%)$ cases of atypical autism, and $27(38 \%)$ cases of Asperger's syndrome. $441(89 \%)$ children were documented as having been assessed by a neurodevelopmental paediatrician, $411(83 \%)$ by a speech therapist, and 422 $(85 \%)$ by a child psychiatrist or a clinical or educational psychologist. 192 (39\%) were recorded as having also been assessed at a centre specialising in autism.

The median ages at diagnosis, first parental concern, and regression according to diagnostic category are shown in table 1 . Age at parental concern showed big peaks at 18 months and 24 months for core and atypical autism. With one exception, the earliest age at diagnosis was 18 months in the core and atypical autism groups and 30 months in the Asperger's syndrome group.

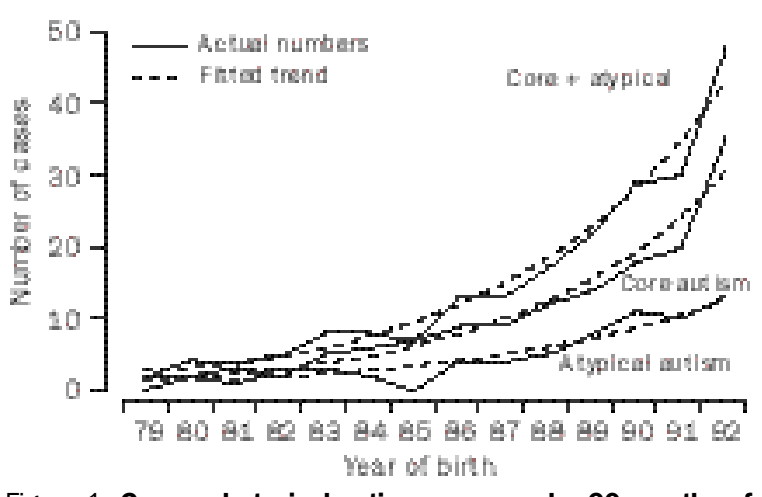

Figure 1: Core and atypical autism cases under 60 months of age and fitted trends by year of birth 1979-92

Regression was recorded for $29 \%$ of core autism cases compared with $18 \%$ of atypical cases and $6 \%$ of those with Asperger's syndrome.

The number of cases by year of birth showed a steady rise peaking in the early to mid 1990s, followed by a sharp decline that was most pronounced for cases of core and atypical autism. This decline is attributable to delays in diagnosis inherent in the disorders. There was a significant upward trend over the period 1979-92 for core and atypical cases (test for zero trend $\mathrm{p}<0 \cdot 001$ ) and a nearly significant upward trend for Asperger's syndrome $(p=0 \cdot 06)$. For the core and atypical cases, there was no evidence of a sudden "step-up" in 1987, the first birth cohorts eligible for MMR vaccine in the second year of life $(p>0 \cdot 25)$. Neither was there evidence that the exponential trend changed after 1987 (figure 1).

A total of 389 children with core autism, atypical autism, or Asperger's syndrome were born after 1987; $336(86 \cdot 4 \%)$ of these had received MMR vaccine by the end of the second year of life and a further $17(4 \cdot 4 \%)$ received the vaccine after this age. The modal age at which MMR vaccine was given was 13 months. The MMR vaccine coverage in the 389 study cases did not differ significantly from that in the same birth cohorts in the North East Thames region as a whole (figure 2). Trends in the incidence of autism by birth cohort since 1987 (figure 1) were not temporally associated with changes in vaccine coverage (figure 2). Owing to the small numbers of Asperger's cases eligible for MMR vaccine in the second year of life (49), and their older age

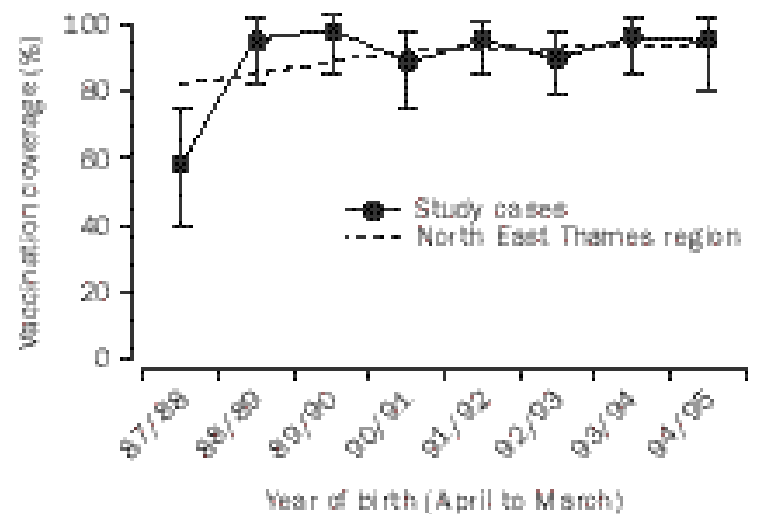

Figure 2: MMR vaccine coverage by second birthday and year of birth

Error bars $=95 \% \mathrm{Cl}$. Coverage figures for birth cohorts in North East Thames obtained from Vaccination and Immunisation Summary Information for 1996-97 produced by Government Statistics Service DH Statistics Division. 


\begin{tabular}{|c|c|c|c|c|}
\hline \multirow[t]{2}{*}{$\begin{array}{l}\text { Event and risk period } \\
\text { (months) }\end{array}$} & \multicolumn{2}{|l|}{ MMR vaccine(s) } & \multicolumn{2}{|l|}{$\begin{array}{l}\text { MIMR, measles, } \\
\text { mumps and rubella } \\
\text { vaccine(s) }\end{array}$} \\
\hline & $\begin{array}{l}\text { Relative } \\
\text { incidence } \\
(95 \% \mathrm{Cl})\end{array}$ & $\begin{array}{l}\text { Number } \\
\text { of } \\
\text { events }\end{array}$ & $\begin{array}{l}\text { Relative } \\
\text { incidence } \\
(95 \% \mathrm{Cl})\end{array}$ & $\begin{array}{l}\text { Number } \\
\text { of } \\
\text { events }\end{array}$ \\
\hline \multicolumn{5}{|c|}{$\overline{\text { Autism diagnosis }(\mathrm{n}=357)}$} \\
\hline$<12$ & $0.94(0.60-1.47)$ & 31 & $0.80(0.53-1.22)$ & 36 \\
\hline$<24$ & $1.09(0.79-1.52)$ & 138 & $1.05(0.76-1.44)$ & 162 \\
\hline \multicolumn{5}{|c|}{ Parental concern $(n=326)$} \\
\hline$<6$ & $1.48(1.04-2.12)$ & 75 & $1.19(0.84-1.69)$ & 82 \\
\hline$<12$ & $0.90(0.63-1.29)$ & 120 & $0.86(0.60-1.23)$ & 142 \\
\hline \multicolumn{5}{|l|}{ Regression ( $n=105$ ) } \\
\hline$<2$ & $0.92(0.38-2 \cdot 21)$ & 7 & $1.24(0.61-2.56)$ & 11 \\
\hline$<4$ & $1.00(0.52-1.95)$ & 17 & $1.31(0.73-2.33)$ & 24 \\
\hline$<6$ & $0.85(0.45-1.60)$ & 28 & $0.99(0.56-1.75)$ & 35 \\
\hline
\end{tabular}

Table 2: Relative incidence and numbers of events in risk periods after vaccination with one or more MMR vaccine or one or more MMR, single-antigen measles and mumps plus rubella vaccines, by event type in children with core or atypical autism

at diagnosis, these cases were not included in further analyses of vaccination status.

Of the 356 cases of core or atypical autism with age at diagnosis of 18 months or greater, 233 received MMR vaccine before this age, 64 never received MMR vaccine, and 59 received MMR vaccine at 18 months or later. There were no differences in age at diagnosis between those vaccinated before or after 18 months of age and those never vaccinated $(p=0 \cdot 41)$ and no interaction between these vaccine categories and year of birth $(p=0 \cdot 29)$. The parameter estimates, expressed as folddifferences in geometric mean ages were: vaccinated before 18 months over unvaccinated 0.91 (95\% CI 0.79-1.05); vaccinated after 18 months over unvaccinated $0.93(0.81-1.08)$.

The results of the case-series analyses are shown in table 2 ; the results were similar when the analysis was restricted to cases confirmed by ICD10 criteria. There was no significant clustering of interval to diagnosis or regression within the time periods defined. There was a significant clustering of parental concern within 6 months of vaccination $(p=0.03)$ but no significant excess risk in any of the other periods investigated $(<1,<2,<3,<4$, $<5,<7,<8,<9,<10,<11$, and $<12$ months after vaccination). The distribution of parental concern by interval in months since latest MMR vaccination showed a peak at 5 months (22 cases compared with a range of four to 14 for the remaining intervals up to 12 months). This excess was largely attributable to the peak recorded age of parental concern being 18 months, combined with the peak in MMR vaccination at 13 months. When the data were reanalysed without cases with recorded age at parental concern of 18 months $(n=61)$, all statistical significance disappeared. For case-series analyses restricted to cases of core autism, the results (not shown) were similar to those in table 2 with the exception of age at onset of parental concern within 6 months of MMR vaccination, which showed no significant excess risk (relative incidence 1.25 [95\% CI $0 \cdot 81-1 \cdot 95]$ ); the relative incidence for atypical cases when analysed separately remained raised at $1.99(1 \cdot 08-3 \cdot 68)$.

\section{Discussion}

Vaccination and vaccine safety are issues of major concern to the public, their elected representatives, and all health-care workers. Possible adverse reactions to vaccines have a particular attraction to various pressure groups and to the media, with important, and possibly catastrophic, effects on public confidence in immunisations and on vaccine uptake. ${ }^{10}$ The study by Wakefield and others ${ }^{1}$ and earlier work from those investigators suggesting an association between measlescontaining vaccines and inflammatory bowel disease $\mathrm{e}^{11,12}$ (not confirmed in their subsequent studies ${ }^{13-15}$ ) received much media attention and have had an adverse effect on immunisation uptake. ${ }^{16}$ The consequences of these events are that many children are now at risk of measles, mumps, and rubella, and that the possibility of eradication of measles has been delayed.

Our study was designed to test the hypothesis that MMR vaccination is causally associated with autism. The study has some limitations: two of these are that we could not verify the diagnosis according to ICD10 criteria in some cases, and that the ascertainment may have been incomplete. The clinical notes were of variable quality and many did not contain systematic or regularly updated information which would have allowed independent validation of the diagnosis, particularly in the children with atypical autism or Asperger's syndrome. However, we have confidence in the overall reliability of the diagnosis of autism in our study. Most cases were documented as having been assessed by specialist clinicians, and the remainder are highly likely to have been as well. There was close similarity between the ICD10-confirmed and non-confirmed cases, and all the analyses showed almost identical results when repeated with only ICD10-confirmed cases. We made substantial efforts to capture all cases of autism in study districts from multiple sources, but inevitably some cases will have been missed, particularly children educated outside their borough and not known to local health services or education authorities. Nevertheless, our prevalence rates for autism are similar to those reported in other contemporary studies. ${ }^{17}$ Incomplete case ascertainment would not affect the validity of our results for the caseseries analyses unless the unidentified children with autism were more likely than those we identified to have had onset in close temporal association with MMR vaccine; this possibility seems unlikely.

There is uncertainty about whether the prevalence of autism is increasing. ${ }^{18}$ Our study is consistent with an increase in the incidence of autism in recent birth cohorts. This increase may be real or a reflection of other factors such as better recording arrangements in recent years, the increasing recognition of higher functioning children with autism and Asperger's syndrome, together with an increasing number of professionals trained to recognise the disorders. However, whether real or artifactual, the trend in increasing incidence with successive birth cohorts to 1992 was not related to the introduction of MMR vaccine or to vaccine coverage, which reached a plateau during a period in which autism incidence was apparently increasing.

We looked for evidence of a possible causal association between MMR vaccination and onset of autism by investigating whether, after adjustment for birth-cohort effects on incidence, age at diagnosis of autism varied with vaccination status. The age at diagnosis was found to be independent of whether MMR vaccine was given, or in those vaccinated, whether the vaccine was given before or after 18 months of age-the earliest age at diagnosis of core or atypical autism. The proportion of 
core and atypical cases vaccinated by the end of the second year of life was similar to that in the same birth cohorts in the North East Thames region. None of these analyses suggest a causal association between MMR vaccination and autism.

The case-series analyses showed no evidence of temporal clustering between MMR or other measlescontaining vaccines and diagnosis of autism. Regression, as reported in other studies, ${ }^{5}$ occurred in nearly a third of the cases of core autism; regression was not clustered in the months after vaccination. For age at first parental concern, no significant temporal clustering was seen for cases of core autism or atypical autism, with the exception of a single interval within 6 months of MMR vaccine associated with a peak in reported age at first parental concern at 18 months. This peak is likely to reflect the difficulty experienced by parents in defining the precise age at onset of symptoms in their child, particularly those with atypical autism, and consequent approximation with preference for 18 months.

Our results do not support the hypothesis that MMR vaccination is causally related to autism, either its initiation or to the onset of regression-the main symptom mentioned in the paper by Wakefield and others. ${ }^{1}$ The data on clinical presentation and immunisation status of the cases in our study were recorded before the recent publicity suggesting a possible link between MMR vaccine and autism. The two datasets were collected independently of each other, so avoiding the bias that can occur when cases are ascertained as a result of a perceived link with vaccination. This study does not rule out the possibility of a rare idiosyncratic response to MMR. However, if such an association occurs, it is so rare that it could not be identified in this large regional sample. Our findings, based on a large study, confirm and extend those of Gillberg and Heijbel, ${ }^{19}$ which showed no evidence of a causal association between MMR vaccine and autistic disorder in Sweden. We hope our results will reassure parents and others who have been concerned about the possibility that MMR vaccine is likely to cause autism and that they will help restore confidence in MMR vaccine.

\section{Contributors}

Brent Taylor, Elizabeth Miller, Christina Petropoulos, and Jun Li were responsible for study design. Brent Taylor, Christina Petropoulos, and Isabelle Favot-Mayaud were responsible for case identification and ascertainment. Paddy Farrington undertook the statistical analyses. Elizabeth Miller, Pauline Waight, Jun Li, Isabelle Favot-Mayaud, and
Brent Taylor were responsible for data handling and processing. All investigators contributed to the writing of the paper.

\section{Acknowledgments}

We thank Nick Andrews (CDSC) for his help with statistical analysis, Andrew Lloyd Evans (neurodevelopmental paediatrician) and Sarah Willenberg (speech therapist) for advice, and Ioanna Kontagianni and Ekundayo Ajani-Obe for help with piloting and data collection. The study was funded by the Medicines Control Agency.

\section{References}

1 Wakefield AJ, Murch SH, Anthony A, et al. Ileal-lymphoid-nodular hyperplasia, non-specific colitis, and pervasive developmental disorder in children. Lancet 1998; 351: 637-41.

2 Chen RT, DeStefano F. Vaccine adverse events: causal or coincidental? Lancet 1998; 351: 611-12.

3 Lee JW, Melgaard B, Clements CJ, Kane M, Mulholland EK, Olivé J-M. Autism, inflammatory bowel disease, and MMR vaccine. Lancet 1998; 351: 905

4 Bedford H, Booy R, Dunn D, et al. Autism, inflammatory bowel disease, and MMR vaccine. Lancet 1998; 351: 907.

5 Siegel B, Pliner C, Eschler J, Elliot G. How children with autism are diagnosed: difficulties in identification of children with multiple developmental delays. F Dev Behav Paediatr 1988; 9: 199-204.

6 Nicholl A, Elliman D, Ross E. MMR vaccination and autism 1998. BMF 1998; 316: 715-16.

7 Farrington CP. Relative incidence estimation from case series for vaccine safety evaluation. Biometrics $1995 ; \mathbf{5 1}: 228-35$.

8 Farrington CP, Nash J, Miller E. Case series analysis of adverse reactions to vaccines: a comparative evaluation. Am f Epidemiol 1996; 143: $1165-73$.

9 Farrington P, Pugh S, Colville A, et al. A new method for active surveillance of diphtheria/tetanus/pertussis and measles/mumps/rubella vaccines. Lancet 1995; 345: 567-69.

10 Gangarosa EJ, Galazka AM, Wolfe CR, et al. Impact of anti-vaccine movements on pertussis control: the untold story. Lancet 1998; 351: 356-61.

11 Wakefield AJ, Pittilo RM, Sim R, et al. Evidence of persistent measles virus infection in Crohn's disease. f Med Virol 1993; 39: 345-53.

12 Thompson NP, Montgomery SM, Pounder RE, Wakefield AJ. Is measles vaccination a risk for inflammatory bowel disease? Lancet 1995; 345: 1071-74.

13 Thompson NP, Pounder RE, Wakefield AJ. Perinatal and childhood risk factors for inflammatory bowel disease: a case control study. Eur $\mathcal{F}$ Gastroenterol Hepatol 1995; 7: 385-90.

14 Morris DL, Montgomery SM, Ebrahim S, Pounder RE, Wakefield AJ. Measles vaccination and inflammatory bowel disease in the 1970 British cohort study. Gut 1997; 41 (suppl 3): 37 (abstr).

15 Chadwick N, Bruce IJ, Schepelmann S, Pounder RE, Wakefield AJ. Measles virus DNA is not detected in inflammatory bowel disease using hybrid capture and reverse transcriptase followed by polymerase chain reaction. $\mathcal{F}$ Med Virol 1998; 55: 305-11.

16 CDSC. COVER/Korner: April to June 1998. Commun Dis Rep CDR Wkly 1998; 8: 345-46.

17 Fombonne E, D Mazaubrun C, Cans S, Grandjean J. Autism and associated medical disorders in a large French epidemiological sample. Am Acad Child Adolesc Psychiatry 1997; 36: 1561-69.

18 Wing L. Autistic spectrum disorders: no evidence for or against an increase in prevalence. $B M F$ 1996; 312: 327-28.

19 Gillberg C, Heijbel H. MMR and autism. Autism 1998; 2: 423-24. 